\title{
Radial Structure in the Preterm Cortex; Persistence of the Preterm Phenotype at Term Equivalent Age?
}

\author{
Andrew Melbourne ${ }^{1}$, Giles S. Kendall ${ }^{2}$, M. Jorge Cardoso ${ }^{1}$, Roxanna Gunney ${ }^{3}$, \\ Nicola J. Robertson ${ }^{2}$, Neil Marlow ${ }^{2}$, and Sebastien Ourselin ${ }^{1}$ \\ 1 Centre for Medical Image Computing, University College London, UK \\ 2 Academic Neonatology, EGA UCL Institute for Women's Health, London, UK \\ ${ }^{3}$ Neuroradiology, Great Ormond Street Hospital for Children NHS Trust, \\ London, UK
}

\begin{abstract}
Preterm birth increases the risk of perinatal brain injury and is believed to initiate a cascade of processes causing white matter damage resulting in subsequent neurological deficit; neonatal magnetic resonance imaging provides a number of potential biomarkers of this deficit. In this work we unify measures of the cortical folding pattern and of white matter integrity to establish correlation between grey and white matter derived properties. Diffusion weighted MRI has revealed that the cortical grey matter in the extremely preterm period exhibits a strong transient radial organisation suggesting neuronal axons are orientated towards the underlying white matter. This effect is lost during cortical maturation and is considered no longer visible on MRI at term equivalent age. Here we show that, in a group of 19 infants, radial organisation in the cortical grey matter remains detectable at term-equivalent age and that there is a strong anterior-posterior asymmetry. A group of three infants with moderate or severe abnormal white matter abnormality have significantly higher cortical grey matter radial organisation $(p<0.02)$, higher grey matter FA $(p<0.01)$ and a lower measure of cortical complexity $(p<0.03)$ than infants with normal or mild abnormal white matter abnormality; all measures associated with the preterm phenotype before term equivalent age. The novel combination of state-of-the-art imaging techniques, analysing grey-matter based spatial characteristics, may provide insight into the mechanism of neurodevelopmental deficits seen in infants with abnormal MR imaging at term equivalent age.
\end{abstract}

\section{Introduction}

Preterm brain injury is increasingly recognised to be an amalgamation of specific brain injury and abnormal brain development [1]. Advances in MR techniques including volumetric and cortical surface analysis [2] have begun to define differences in the brains of preterm infants imaged at term compared to term born controls and thus can help define biomarkers for prediction of subsequent neurodevelopment outcome. This preterm brain phenotype extends beyond reduction in volume and anisotropy of the cortical white matter to encompass decreased volume in the cortical grey matter, basal ganglia and cerebellum. 
Very preterm birth coincides with a period of rapid brain growth and development [1]. During this period there is proliferation and migration of neurones from the subventricular zone to the cortex by radial migration. This results in radial cortical organisation; axons within the cortex lie perpendicular to the cortex, and this appears to be observed using diffusion tensor imaging as highly organised directional diffusion with consequent high fractional anisotropy. Examination of the principal eigenvectors suggest that axons are orientated perpendicular to the cortical surface towards the underlying white matter 34. During normal cortical maturation the progressive development of multiple associative cortical interconnections results in a loss of this diffusion pattern by 35 weeks post menstrual age 3. In addition, measures of gyrification are found to correlate with the underlying grey matter diffusion properties [5], although not after adjustment for gestational age at birth.

As preterm white matter injury is associated with a reduction in cortical grey matter volume we hypothesise it may also be associated with abnormal cortical development and organisation including higher cortical fractional anisotropy, lower white matter fractional anisotropy and the continued presence of cortical radial organisation visible on MRI at term equivalent age. In addition, measures of gyrification are found to correlate with the underlying grey matter diffusion properties [5]. We therefore also aim to correlate these findings to measures of cortical folding in babies with and without white matter injury on conventional MR imaging. Here, we develop a novel algorithm to specifically detect residual radial structure in the preterm cortex and show that its presence may be significantly higher in infants with moderate or severe white matter abnormality. The algorithm uses state-of-the-art image analysis techniques to develop, to the best of our knowledge, the first description of radial cortical architecture in very preterm infants at term equivalent age. Addressing this neonate-specific neuroscience question using such a technique may provide insight into the mechanism of neurodevelopmental deficits seen in infants with abnormal MR imaging at term equivalent age.

\section{Methods}

In this section we describe the steps needed to form the algorithm used for radial structure detection, comprising image segmentation, cortical surface extraction and diffusion parameter estimation and provide details of the 19 subject cohort of preterm infants scanned at term equivalent age.

\subsection{Segmentation and Cortical Surface Analysis}

Segmentation of the grey and white matter components of each infant is carried out using an adaptive neonatal-specific segmentation algorithm $[$ [ 6 . The segmentation method estimates the cortical grey and white matter regions within an

\footnotetext{
${ }^{1}$ Available as part of the niftyseg package at: sourceforge.net/projects/niftyseg/
} 
expectation maximisation routine whilst simultaneously spatially relaxing priors over the tissue classes. The algorithm incorporates a Markov random field for noise reduction, automated bias field correction and an implicit partial volume strategy. The interface between the segmented grey and white matter is found using a level-set technique, extracting a function at the surface upon which the gradient and curvature are implicitly defined at every position [7]. The boundary between grey and white matter is optimised using an evolving level set (1);

$$
\frac{\delta \phi}{\delta t}+\left(\lambda_{s} s(\mathbf{x}, t)-\lambda_{c} c(\mathbf{x}, t)\right)\|\nabla \phi(\mathbf{x}, t)\|=0
$$

solving a partial differential equation for the function $\phi(\mathbf{x}, t)$ combining a signed distance function from the boundary, $s$, and a restriction on the curvature of the resulting function, $c$, with scalar weighting parameters $\lambda_{s}=0.1$ and $\lambda_{c}=0.4$ respectively. The level set is initialised along the white matter boundary and the result is a map of signed distances from the optimised GM/WM boundary.

We define measures of the gradient and curvature on this implicit surface, similar to the method used in [7]. The gradient of the level set is defined as perpendicular to the surface whilst the local Hessian matrix of second order derivatives can be use to define two curvatures; the eigenvalues of the local Hessian matrix define the principal curvatures, $\kappa_{1}$ and $\kappa_{2}$ (with $\left.\kappa_{1}>\kappa_{2}\right)$ which summarise the local shape. Explicitly we define the signed shape index, $S$ (2), describing how cup-like or saddle-like the surface is $[8]$.

$$
S=\frac{2}{\pi} \tan ^{-1}\left(\frac{\kappa_{2}+\kappa_{1}}{\kappa_{1}-\kappa_{2}}\right)
$$

The distribution of the absolute values of shape index found on the surface may be summarised by the standard deviation of the absolute values of the shape index (implying that gyri and sulci are indistinguishable). Briefly, if the cortex has a complicated folding pattern, this is likely to introduce a larger range of possible shape-index values, and thus the standard deviation of this distribution, $\sigma_{S}$, will also be high. In contrast to global or slice-wise measures like the gyrification index, defined either automatically or manually [95], the shape index is defined at every position on the cortex, thus the standard deviation statistic may be defined over any arbitrary region.

\subsection{Diffusion Imaging and Registration}

Local fibre orientation in each voxel of the cortical grey matter is estimated by fitting the probabilistic ball and (two) stick model [10], thus each voxel contains a measure of its diffusivity and an estimate of the principal diffusion direction used to infer the underlying fibre orientation. Mean diffusivity images are registered to a single baseline and the diffusion information is then combined with the segmentation result by affine registration between the T1w image and the mean diffusivity image. Subsequently the GM, WM and level set segmentations are propagated to the diffusion space and we define the fractional anisotropy (FA) image in order to investigate spatial and group trends in the GM and WM FA. 


\subsection{Radial Structure Detection}

After registration between the level set and the diffusion images, the radial direction perpendicular to the cortex in the diffusion space is defined by the gradient of the level set, $g$ (Equation 3). If, at any position, the principal diffusion direction [10] in the grey matter, $p$, is parallel to the gradient of the level set function, the structure of the cortical grey matter can be considered radial (Equation 3). This measure of radial structure should not be confused with the radial component of the directional diffusivity given by the sum of the second and third eigenvalues of the diffusion tensor which has been shown to correlate with gestational age at birth [5]. Figure 1; illustrates the technique, relating the principal diffusion direction found using the ball and stick model to the direction across the underlying GM/WM boundary.

$$
d=|\hat{g} \cdot \hat{p}|
$$

Integrating this function over the entire cortex results in a summary measure, $D$, of the radial structure for each infant, Equation 4 in spherical polar coordinate:2.

$$
D=\int_{-\pi}^{\pi} \int_{0}^{\pi} \int_{0}^{\infty}|\hat{g} \cdot \hat{p}| r^{2} \sin \phi \delta r \delta \phi \delta \theta
$$

The measure, $D$, may also be subdivided into segments, $\delta \theta$ in the axial plane, $\theta(D(\theta, \delta \theta))$, to observe any intra-subject spatial relationship along the medial lateral and anterior-posterior directions. This direction is expected to show the major trends in the folding pattern [9] and be sensitive to the known pattern of white matter myelination [11. In any region of the cortex, $D(\theta, \delta \theta)$ may be compared with the underlying average grey and white matter FA and also to the standard deviation of absolute shape index values as described above, thus any observed radial structure can be correlated with known measures of cortical grey and white matter maturation. Visualisation of this information is provided using axially orientated rose plots; data is divided into segments and summary values found over each segment is presented by angle in the axial plane whilst the radius at any point represents the magnitude of the associated parameter. This style of visualisation allows variation in the observed parameters to be contrasted in the anterior-posterior and medial-lateral directions.

\subsection{Data}

Twenty infants born very preterm (less than 32 weeks completed gestation) underwent an MRI at term equivalent age. High-resolution T1-weighted imaging $(0.39 \times 0.39 \times 1 \mathrm{~mm})$ and $2 \times 30$ directional diffusion tensor imaging $(b=$ $600 \mathrm{~s} . \mathrm{mm}^{-2}$ at $0.9 \times 0.9 \times 3 \mathrm{~mm}$ ) were acquired alongside two unweighted images

\footnotetext{
${ }^{2}$ Analytically, the expected value of the radial structure measure $D$ over any arbitrary region containing an isotropic random distribution of grey matter diffusion directions is $D=0.5$. Interestingly, this result is a 3D extension of the classic Buffon's needle problem.
} 

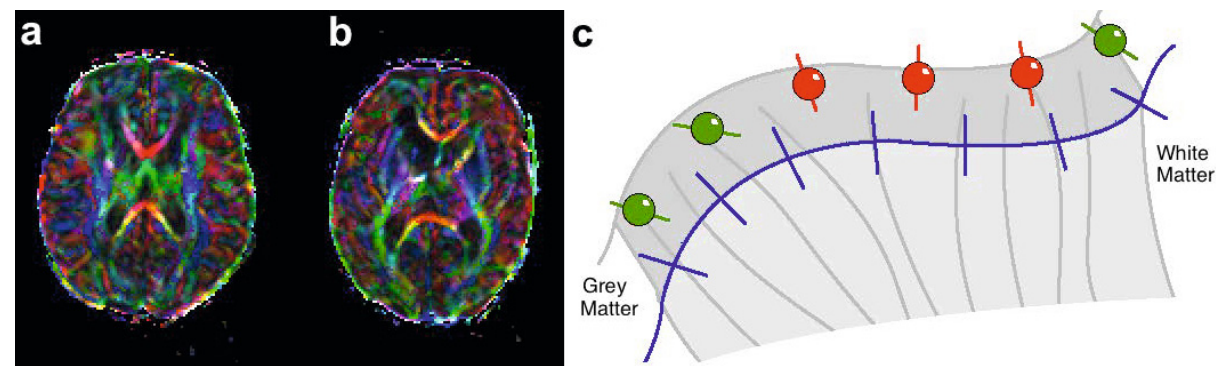

Fig. 1. Axial colour-coded FA slices for a) normal white matter appearance and b) moderate white matter abnormality visible on DTI. Note the suggestion of radially (perpendicular to the cortex) organised structure in the prefrontal cortex in b). c) illustrates the relationship between the principal diffusion direction found using the ball and stick model and the direction of the underlying GM/WM boundary.

and clinical T2 weighted images (3mm slices). One infant was subsequently removed from the study due to the presence of motion artefacts in the diffusion weighted sequence. Conventional MRI was assessed for WM abnormality using an established qualitative scoring system based on a grading system of 5 scales [12. Images were scored by an experienced neuroradiologist blinded to clinical history and neurodevelopmental outcome. WM abnormality was classified as normal/mild or moderate/severe [12.

\section{Results}

Of the 19 babies analysed 11 were males, the average gestation was 25.8 weeks (range 22.9-30.7) with average birthweight of $822 \mathrm{~g}$ (range 447-1185g). MR imaging was obtained at 40.8 weeks post menstrual age (range 38.3-44 weeks). Three babies were classified as moderate / severe white matter injury. The remaining 16 babies were classified as normal / mild using an established white matter scoring system [12.

Figure 1 1 shows colour-coded FA maps for four subjects, red colour coding corresponds to medial-lateral principal diffusion direction (PDD), green to anteriorposterior PDD and blue to superior-inferior PDD; pixel intensity represents the FA value. Figure 17a shows one infant with normal white matter appearance and little visible radial grey matter organisation. Figure 1b shows one infant with moderate white matter abnormality. The radial detection measure is calculated for the 16 infants with normal /mild white matter abnormality and for the 3 infants with moderate/severe white matter abnormality. Figure 2 illustrates the change in cortical grey and white matter properties with angle in the axial plane for the normal/mild group when divided into 64 equal angle segments (the anterior-posterior direction is aligned along the $90-270^{\circ}$ axis). Figure $2 \mathrm{a}$ measures the radial organisation (Equation 3) showing increased radiality in the frontal and pre-frontal region relative to the occipital region even at term equivalent age. In addition the pattern is broadly symmetric along the mediallateral axis. Figure $2 \mathrm{~b}$ and $\mathrm{c}$ illustrate the corresponding average cortical grey 
a)
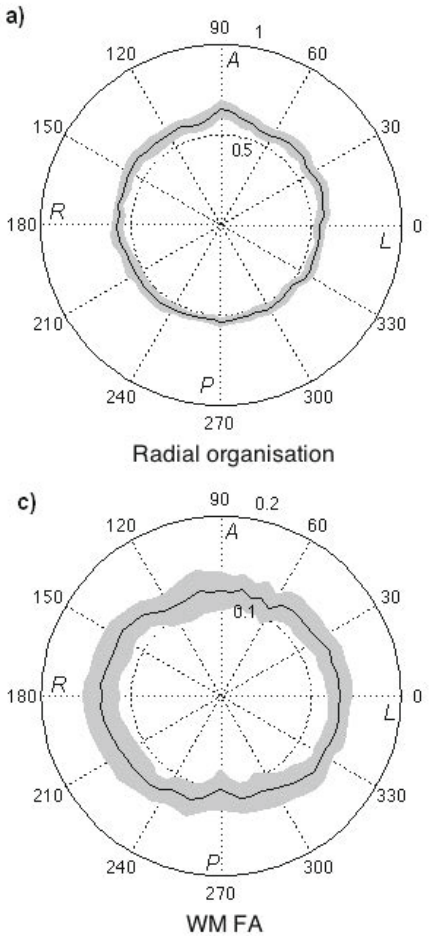

b)
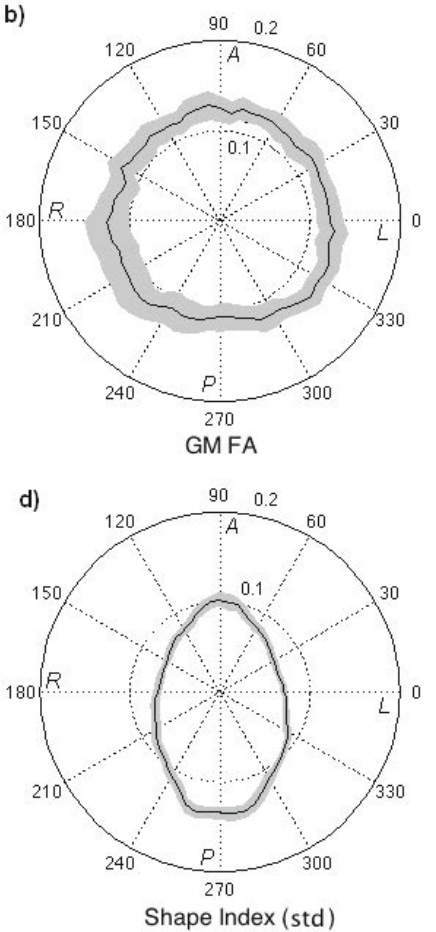

Fig. 2. Rose plots of each parameter with angle in the axial plane (normal/mild white matter abnormality group only): a) radial organisation measure; b) average GM FA value; c) average WM FA value (with each axial segment) and d) absolute shape index standard deviation. All plots show the mean in black, the grey region is $\pm \sigma$.

and underlying white matter FA values (calculated using the propagated segmentations) for each segment. The average grey matter FA is broadly isotropic around the cortex and appears higher in the pre-frontal region relative to the occipital region. The corresponding white matter pattern suggests higher FA along the medial-lateral axis, possibly representing the partial myelination of the corticospinal tracts. Total average grey and white matter FA are negatively correlated $(r=-0.56, p<0.02)$, which concords with the expected trajectories of white and grey matter maturation; the average FA associated particularly with the corticospinal tracts increases with gestation age whilst the observed grey matter FA reduces as a result of increased cortical inter-connectivity [5]. Figure $2 \mathrm{~d}$ plots the standard deviation of the shape index values found in each region of the cortex, representing a measure of the cortical folding pattern. The cortical folding pattern is more complicated posterior than anterior. The total shape index standard deviation has a non-significant negative correlation with the grey matter FA $(r=-0.30, p=0.22)$.

Figure $3 \mathrm{a}$ and $\mathrm{b}$ show the spatial radial structure measure (Equation $3, \mu \pm \sigma$ ) for the normal/mild and moderate/severe white matter abnormality groups 

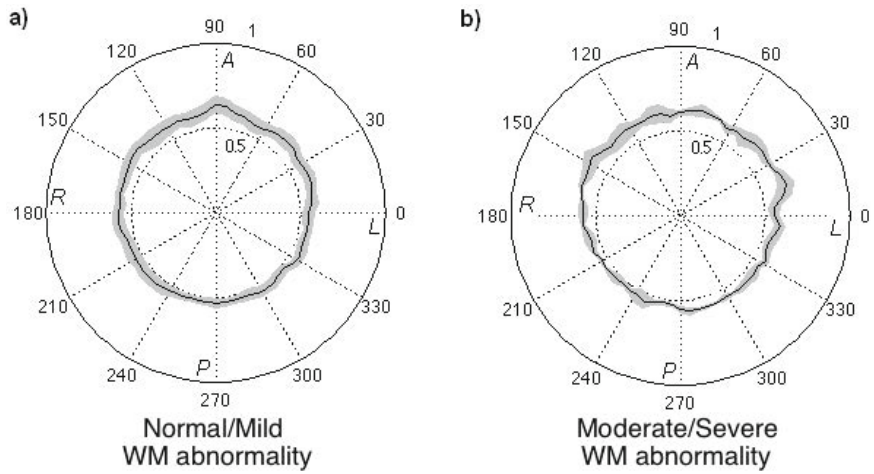

Fig. 3. Rose plots of radial structure for: a) normal or mild white matter abnormality $(\mathrm{n}=16)$ and $\mathrm{b})$ moderate or severe white matter abnormality $(\mathrm{n}=3)$. All plots show the mean in black, the grey region is $\pm \sigma$. The value of $D$ (Equation 4) is found to be significantly higher in the moderate/severe white matter abnormality group than the normal/mild group $(p<0.02)$, although the group sizes are disparate.

respectively. The moderate/severe group has a significantly higher measure of radial structure $(D$, Equation (4) than does the normal/mild group $(p<0.02)$, although the correlation is low. Additionally, the moderate/severe white matter abnormality group has significantly higher grey matter FA $(p<0.01)$ and significantly lower shape index standard deviation $(p<0.03)$ than infants with normal/mild white matter abnormality. Each of these features is associated with the preterm phenotype before term equivalent age, although it is not clear which is most informative.

\section{Discussion}

This work has used a combined analysis of segmentation and diffusion properties in both the grey and white matter to suggest a link between white matter abnormality on conventional MRI and structural organisation in very preterm infants scanned at term equivalent age. White and grey matter diffusion properties correlate with structural cortical folding information and retention of the preterm radial cortical architecture at term equivalent age in a small group of babies with moderate to severe white matter abnormality, particularly in the prefrontal cortex. The presence of this radial organisation in the cortex at term equivalent age may suggest that moderate to severe white matter abnormality is associated with a delay or disruption in normal cortical maturation, although we emphasise that the small group size makes general inference difficult. To our knowledge this is the first description of radial cortical architecture in very preterm infants at term equivalent age and may provide insight into the mechanism of neurodevelopmental deficits seen in infants with abnormal MR imaging at term equivalent age. Further work with serial MR acquisitions through the preterm period and beyond in combination with long term neurodevelopmental followup may help identify the nature of these changes and their association with 
outcomes in childhood. Furthermore, formalisation of this technique into a robust analysis of grey-matter based spatial statistics may be a useful complement to existing white-matter techniques.

Acknowledgements. We would like to acknowledge UK registered charity SPARKS, the National Institute for Health Research (NIHR), the Fundação para a Ciência e a Tecnologia, Portugal, the EPSRC (EP/H046410/1) and the Comprehensive Biomedical Research Centre (CBRC) Strategic Investment Award (Ref. 168).

\section{References}

1. Volpe, J.J.: Brain injury in premature infants: a complex amalgam of destructive and developmental disturbances. Lancet Neurol. 8(1), 110-124 (2009)

2. Boardman, J.P., Craven, C., Valappil, S., Counsell, S.J., Dyet, L.E., Rueckert, D., Aljabar, P., Rutherford, M.A., Chew, A.T.M., Allsop, J.M., Cowan, F., Edwards, A.D.: A common neonatal image phenotype predicts adverse neurodevelopmental outcome in children born preterm. Neuroimage 52(2), 409-414 (2010)

3. McKinstry, R.C., Mathur, A., Miller, J.H., Ozcan, A., Snyder, A.Z., Schefft, G.L., Almli, C.R., Shiran, S.I., Conturo, T.E., Neil, J.J.: Radial organization of developing preterm human cerebral cortex revealed by non-invasive water diffusion anisotropy mri. Cereb. Cortex 12(12), 1237-1243 (2002)

4. Adams, E., Chau, V., Poskitt, K.J., Grunau, R.E., Synnes, A., Miller, S.P.: Tractography-based quantitation of corticospinal tract development in premature newborns. J. Pediatr. 156(6), 882-888, 888.e1 (2010)

5. Deipolyi, A.R., Mukherjee, P., Gill, K., Henry, R.G., Partridge, S.C., Veeraraghavan, S., Jin, H., Lu, Y., Miller, S.P., Ferriero, D.M., Vigneron, D.B., Barkovich, A.J.: Comparing microstructural and macrostructural development of the cerebral cortex in premature newborns: diffusion tensor imaging versus cortical gyration. Neuroimage 27(3), 579-586 (2005)

6. Cardoso, M.J., Melbourne, A., Kendall, G.S., Modat, M., Hagmann, C.F., Robertson, N.J., Marlow, N., Ourselin, S.: Adaptive Neonate Brain Segmentation. In: Fichtinger, G., Martel, A., Peters, T. (eds.) MICCAI 2011, Part III. LNCS, vol. 6893, pp. 378-386. Springer, Heidelberg (2011)

7. Awate, S.P., Yushkevich, P.A., Song, Z., Licht, D.J., Gee, J.C.: Cerebral cortical folding analysis with multivariate modeling and testing: Studies on gender differences and neonatal development. Neuroimage 53(2), 450-459 (2010)

8. Koenderink, J.J., van Doorn, A.J.: Surface shape and curvature scales. Image and Vision Computing 10(8), 557-564 (1992)

9. Zilles, K., Armstrong, E., Schleicher, A., Kretschmann, H.J.: The human pattern of gyrification in the cerebral cortex. Anat. Embryol (Berl.) 179(2), 173-179 (1988)

10. Behrens, T.E.J., Woolrich, M.W., Jenkinson, M., Johansen-Berg, H., Nunes, R.G., Clare, S., Matthews, P.M., Brady, J.M., Smith, S.M.: Characterization and propagation of uncertainty in diffusion-weighted mr imaging. Magn. Reson. Med. 50(5), 1077-1088 (2003)

11. Brody, B.A., Kinney, H.C., Kloman, A.S., Gilles, F.H.: Sequence of central nervous system myelination in human infancy. i. an autopsy study of myelination. J. Neuropathol. Exp. Neurol. 46(3), 283-301 (1987)

12. Woodward, L.J., Anderson, P.J., Austin, N.C., Howard, K., Inder, T.E.: Neonatal mri to predict neurodevelopmental outcomes in preterm infants. N. Engl. J. Med. 355(7), 685-694 (2006) 